\title{
Testing for Dynamic Software Update: An Object-State-Oriented Approach
}

\author{
Di Huang \\ State Key Laboratory for Novel \\ Software Technology, Nanjing \\ University \\ Nanjing, JiangSu, China \\ huangdi@smail.nju.edu.cn
}

\author{
Zelin Zhao \\ State Key Laboratory for Novel \\ Software Technology, Nanjing \\ University \\ Nanjing, JiangSu, China \\ zelinzhao1105@gmail.com
}

\author{
Xiaoxing Ma \\ State Key Laboratory for Novel \\ Software Technology, Nanjing \\ University \\ Nanjing, JiangSu, China \\ xxm@nju.edu.cn
}

\begin{abstract}
Dynamic software update (DSU) can patch programs without stopping them. The updating process includes replacing changed code, transforming stale objects with object transformers, and resuming the execution of the updated program. However, flawed object transformers currently hinder DSU from the wide application, since they may introduce the inconsistencies between the transformed objects with expected new ones, that are created by the new program executing from scratch with the same inputs. To detect such inconsistencies, our approach first utilizes fuzzing testing to explore test inputs, then executes them over the old and new versions of a program within our specially designed parallel executor. Any inconsistency and the corresponding test will be issued. The evaluation over default transformer in 50 updates (14 of them have the inconsistency problem) showed that our approach discovered inconsistency in 16 updates, with 5 false positives and 3 false negatives. We also optimized the seed selection strategy in fuzzing process and improved the efficiency by $25.0 \%$.
\end{abstract}

\section{CCS CONCEPTS}

- Software and its engineering $\rightarrow$ Software evolution; Software testing and debugging.

\section{KEYWORDS}

dynamic software update, fuzzing testing, object state transformer

\section{ACM Reference Format:}

Di Huang, Zelin Zhao, and Xiaoxing Ma. 2021. Testing for Dynamic Software Update: An Object-State-Oriented Approach. In 12th Asia-Pacific Symposium on Internetware (Internetware'20), May 12-14, 2021, Singapore, Singapore. ACM, New York, NY, USA, 10 pages. https://doi.org/10.1145/3457913.3457942

\section{INTRODUCTION}

Software is updated frequently to fix bugs or add new features. Traditionally, the updates are applied with the stop-and-restart strategy, i.e., firstly terminate the running program, deploy the new version program, and finally restart the updated program. Such update schema will inevitably incur service interruptions, which is usually

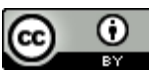

This work is licensed under a Creative Commons Attribution International 4.0 License.

Internetware'20, May 12-14, 2021, Singapore, Singapore

(C) 2021 Copyright held by the owner/author(s)

ACM ISBN 978-1-4503-8819-1/21/05.

https://doi.org/10.1145/3457913.3457942 unacceptable for server programs [2] and will cause serious consequence for mission-critical systems such as stock trading system and traffic controlling system.

Dynamic software update (DSU) was proposed to alleviate such a problem by upgrading programs on-the-fly. DSU systems [10, 22] are responsible for replacing code and more importantly, transforming the stale objects $[13,16,20]$ with object transformers to make them compatible with the new version code. Otherwise, the accesses from the new version code to those stale objects may cause errors or even crash the program.

Therefore, object transformer is a key factor for a safe DSU. A flawed one may cause the inconsistency problem, i.e., the state of a transformed object is not consistent with that of the expected new one, which is created by the new version program executing from scratch with the same input. Taking the Tomcat server as an example, a Request instance, which can be reused between the messages, should reset its state after finishing a response. However, the buggy code in old version recycle method did not reset its state, and the new version program changes the method body of recycle then fixes the bug. The correct transformer for the update should empty the state of old Request instances. Directly copying the old version value to new version object is semantically incorrect and will cause the inconsistency problem.

Default transformer is a trivial but the most widely adopted strategy in existing DSU systems [10, 17, 22]. It only contains two simple rules, inheriting the old values of unchanged fields and assigning default values to new fields. In the previous example, the default transformer will inherit the old state of Request instances since their fields are not explicitly changed, which could lead to unexpected consequences. Existing work $[10,22]$ also suggests developers should manually prepare the object transformer, even though it is an error-prone task. TOS [17] and AOTES [11] are two automatic approaches for transformer synthesis, but they often generate flawed transformers, or fail and fall back to the default transformer.

Therefore, the consistency examination of an object transformer is necessary. However, existing approaches $[1][4,8,12]$ only check the program behavior inconsistency $[6,7,21,24]$ between two programs, such as throwing different exceptions, returning different values, or different resource consumption. In the scope of DSU, the inconsistency focuses on the object states.

In this paper, we proposed an approach to help developers test object transformers. Our approach first employs fuzzing testing [5, $15,18,19,25]$ with an improved seed selection strategy to generate test cases. Then the parallel executor simultaneously drives 


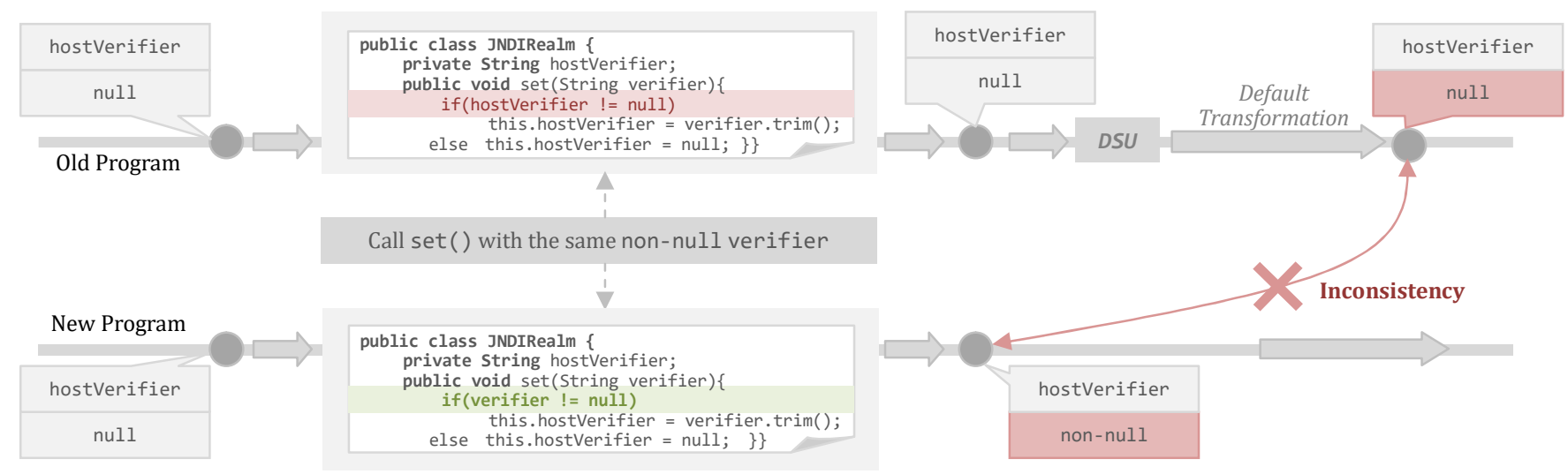

Figure 1: A non-null verifier will trigger the inconsistency of default transformer for commit 7a2eb9 in Tomcat8. Gray arrow means program execution, circle means an object of JNDIRealm class, and the square with "DSU" inside means starting the update process.

two versions of the program with a test, then pauses them at the corresponding point, applies the object transformer, and finally checks the consistency between the transformed objects and the new version ones. Any inconsistency and the corresponding test will be issued to developers. Moreover, the test inputs, which cause the inconsistency achieving a new coverage, will be put into the seeding pool for the next round of fuzzing. Apart from the object state inconsistency, our approach also reports program behavior inconsistency, including throwing different exceptions and returning different values.

We implement the tool and evaluate it on 50 updates from Tomcat server programs. We only test the default transformer since it is always available and being widely adopted. For each update, we first manually examined the consistency of the default transformer and found that 14 updates have the inconsistency problem and 36 do not. To validate the effectiveness, we execute our approach for 1 hour. The results show that our approach detects the inconsistency in 16 updates, with 5 false positives and 3 false negatives.

To evaluate the efficiency of the specially designed coverage guidance in the fuzzing process, we also implemented a variant of our approach that only uses the default guidance strategy. Results showed that this default one got the same accuracy as our approach. However, the default one can only generate 6.28 inputs triggering the inconsistency per second while our approach can generate 7.85 , i.e., the efficiency of the fuzzing process is improved by $25.0 \%$.

The rest of the paper is organized as follows. Section 2 introduces the background of our problem and Section 3 illustrates the approach overview and implementations. Section 4 shows the experimental evaluation of our approach. Before concluding the paper in Section 6, we have some further discussions in Section 5.

\section{BACKGROUND AND MOTIVATION}

\subsection{Dynamic Software Update}

The typical process of DSU is that, while a new version of the program is ready, developers also need to provide the object transformers. Then they request DSU by sending the object transformers along with the code patch to the underlying DSU system. After the request being received, the DSU system starts monitoring the execution of the old version program and pauses it at a safe point to apply the update. The changed code will be replaced, and stale objects will be transformed by calling those object transformers.

Since replacing code has been well solved, the generation and verification of object transformers become the key factors for a safe DSU. Default transformer is a simple solution that only copies the old value to the new object for unchanged fields, and initializes the default value (i.e., null for Object, 0 for int) for changed fields. TOS [17] is a programming-by-example approach that takes old and new version objects as input and synthesizes object transformers from a set of predefined rules. AOTES [11] first synthesizes a method history that can create the current state of a stale object, then replays the history on the new version program to create the corresponding new object. Both TOS and AOTES fall back to the default transformer if they fail to generate one.

\subsection{Motivating Example}

Figure 1 shows a real update that only changes an if condition. In the old version, an object of JNDIRealm class would be initialized with hostVerifier field holding null, and its value should be assigned via calling the set method. However, the developers mistakenly wrote the if condition, which results in the hostVerifier field can not be correctly assigned with a value and is always null. The incorrect if condition was fixed in the new version and hostVerifier can get a value if the verifier parameter is non-null.

This update is tricky since no transformer is capable of handling the hostVerifier field. Transformers are supposed to compute a value based on the old version object and assign it to the new field. The old object in our example has no enough information for the transformation, i.e., hostVerifier is always null. Default transformer only copies the null to the new version object. TOS may generate an over-fitting transformer that conditionally assigns the verifier values collected from input examples, which may make things worse: the null value only makes the program throw 
exceptions, however, the erroneous values may cause unexpected program behaviors. AOTES can not search correct method histories for some old objects, since the execution of set method lost its effect (hostVerifier remains null).

One way to handle this update is recording the verifier parameter in set method for each old JNDIRealm object, and then calling the new set method with the recorded value as the parameter. However, recording the execution history of the old version program is unbearable for most software systems, and always requires a great deal of refactoring to the original program. Therefore we argue that such a case should be handled manually and some transformer examinations should be performed to warn developers about that.

\subsection{Inconsistencies in DSU}

Most updates in DSU are evolutionary maintenance, which usually involve minor changes in code and object states, like the update in Figure 1. Therefore, object transformers are often required to handle the changes in object states to make them compatible with the new version program.

However, a flawed object transformer may cause the state inconsistency problem. We found some simple but very effective heuristics that can be used to check the inconsistency problem for a given transformer. In Figure 1, when two version programs are executed from scratch with the same inputs, the transformed object, should be state consistent with the corresponding new object.

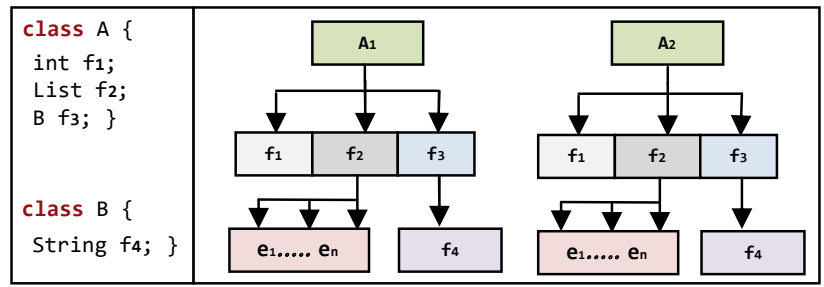

Figure 2: State consistency between two objects

To define state consistency, we view the state of an object as a directed graph (Fig. 2), where nodes are values or variables inside the heap, and directed edges mean the references from one node to another. Comparison of object states is the procedure of traversing the two graphs and comparing each matched nodes. There are no mismatched nodes in the two object graphs because they have the same type. Nodes holding the primitive values are compared directly, with the sub-graphs of other reference nodes considered.

Testing an object transformer is to examine whether any inconsistency problem will be caused. Existing approaches that check the inconsistency between two versions of the program are not suitable for the DSU scenario. They usually focus on the program behavior inconsistency like throwing exceptions, returning values, or resource consumption. Whereas in DSU, the behavior inconsistency is issued to developers, as an assistance for their further review. The inconsistency between object states shows more importance in the transformer preparation, which explicitly indicates the imperfection of the object transformers. Therefore, we test a given object transformer mainly by checking the state consistency with the previously mentioned heuristics.

\section{APPROACH}

We proposed an approach to help developers examine the inconsistency problem for an object transformer, e.g. the default transformer or a manually provided one. DSU developers pay more attention to those updates modifying the declaration of the field, since such updates can directly change the object state and are more likely to cause the inconsistency problem. However, some special updates only changing the method body may still cause the inconsistency problem, which are easily ignored by developers since the fields are not explicitly changed. Therefore, our approach focuses on these special updates that only change the method body.

There are some challenges in testing object transformers, and the most difficult one is the generation of different object states. Systematic test cases could be ideal choices, however, there are no sufficient systematic test cases for each class. We observed that, for our target updates, most inconsistency problems can be triggered by the execution of the changed method. Therefore, we first employ the fuzzing testing to randomly simulate different but valid object states for the changed class, then invoke the changed method with those simulated object states, and apply the given transformer on the old version objects to obtain the transformed ones. Finally, we check the consistency between the transformed objects and the new version objects. Moreover, the behavior consistency is also examined.

The challenges of designing and implementing our approach including:

(1) sufficiently and efficiently construct the input parameters for a method;

(2) simultaneously execute the two versions of a program under the same input parameters;

(3) stably apply the given transformer on the old version object.

\subsection{Overview}

Figure 3 shows the overview of our approach, which mainly includes three steps. First, step a) generates objects of the changed class and input parameters for a given method. Our approach automatically creates a state builder according to the target type. Each state builder contains many random operations and can generate the random state for instances of the target type. Developers can also contribute to the state builder based on their domain knowledge to improve the efficiency of constructing valid states. The two-version test file is generated automatically, which contains the test framework and can simultaneously invoke two versions of a method, apply the transformer on the old version objects, and finally, compare the equivalence of object states.

Once we obtain the state builder and two-version test file, we call the fuzzer to generate new test inputs (step $b$ in Figure 3). We implement our approach based on an open-source fuzzing tool JQFZest $[18,19]$. This tool provides a framework for fuzzing testing for Java programs. To adopt this tool inside our approach, we improve it and add the following functionalities to cooperate with the twoversion test file: parallel executor, transformation simulator, and consistency checker. 


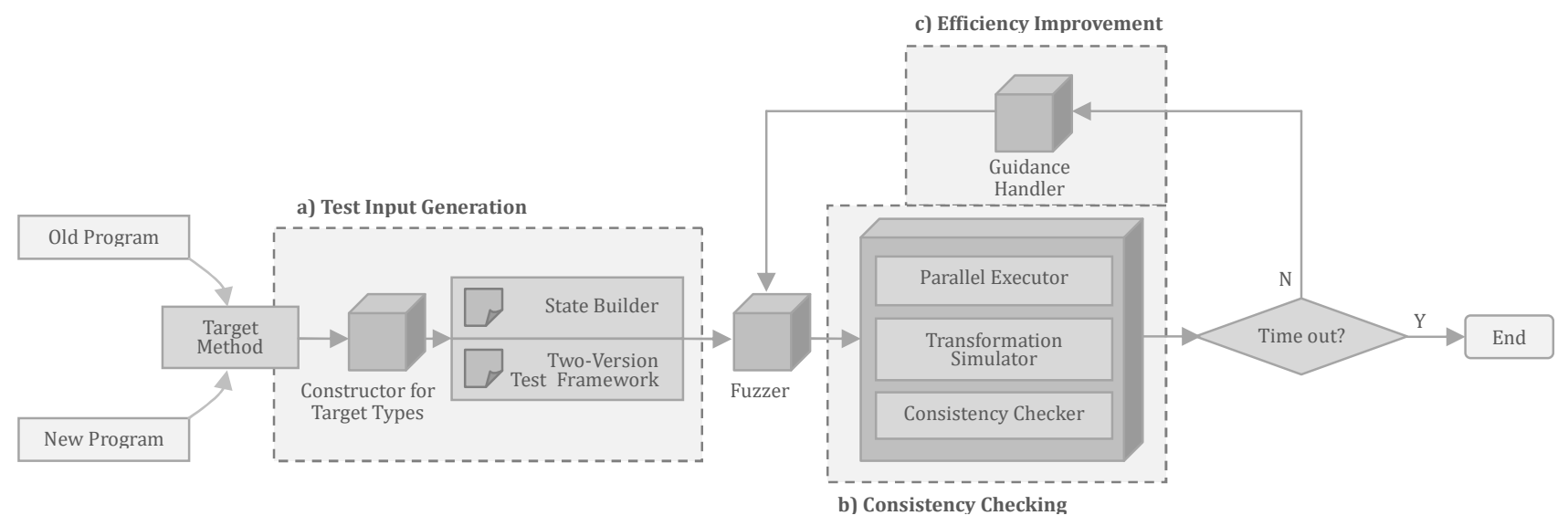

Figure 3: Implementation of Our Tool

We also designed a new coverage guidance in the JQF fuzzer. Once an input triggering the state inconsistency achieves a new coverage, it will be added to the seed pool of fuzzer (step $c$ in Figure 3).

\subsection{Test Input}

\begin{tabular}{|c|l|}
\hline Method & public static void m(Integer value) $\{\ldots . . .\}$. \\
\hline Parameter & Integer value \\
\hline State Builder & $\begin{array}{r}\text { public Integer IntegerBuilder }(\ldots \text { random })\{ \\
\text { return random.nextInt }(-100,100) ;\}\end{array}$ \\
\hline
\end{tabular}

Figure 4: State Builder

Generally, test input refers to the execution path and input parameters. In our approach, we regard the execution path as a target method, since we can simulate the object states and simplify the systematic testing to the unit testing. Thus, the test input mainly refers to input parameters, which are necessary to invoke a single method.

State builder can help us simulate the state of input parameters. Each parameter should have a state builder, which contains many random operations and can assign a random state to the object. As shown in Figure 4, before calling the method m, our tool will generate a random Integer value by using its state builder.

Those state builders can be provided by automatic generation and human effort. To generate the state builder automatically, our tool takes the following steps:

1) Our tool views an object state as a directed graph (Fig. 2), and creates the default assignment statements for each node. For example, a statement random. nextInt $(-100,100)$ will be created automatically for an Integer node. For collections and other reference types, our tool will generate the statements for each element and field, then organize them hierarchically. Moreover, our tool allows developers to control the depth of the directed graph to avoid infinite loops.
2) Since the minor change occurs in the method body, a large number of nodes are irrelevant to this change. For instance, a method receives an object with a complex state, but only reads a single value of its field. In that case, we do not need to consider all the nodes. Our tool employs Javassist [9] library to build a Java call graph between fields and methods, then excludes all irrelevant nodes when generating the state builder.

3) To improve the reliability of automatically-generated state builder, our tool also makes use of the constants contained in Java files. These special constants will be added in the state builder, and each of them will be randomly chosen with a given probability. Our tool uses Javaparser [14] to extract these special constants.

Apart from the automatic generation, our tool allows DSU developers to write and modify the state builder manually. The fuzzer will take the state builder as the original input, then randomly generate the object state.

\subsection{Parallel Executor}

Our tool runs the target method on two versions of the program simultaneously. There are two key points in this implementation: 1) how to invoke the method on two programs, 2) how to manage two programs so that two programs can be executed separately.

For the first question, our tool provides a two-version test file guiding the whole test procedure. Once it obtains the randomlygenerated parameters, it will call the target method on two version programs, with the parameters of the same states. We realize it by using Java reflection.

For the second question, our tool uses two separate classloaders to load two version programs, which are incompatible with each other. Therefore, we can improve the independence of each execution to some extent. However, there remain some defects when two version programs read and write the same local files. In that case, it is meaningless to check the state consistency since we do not need to transform the local files, and this type of change should be manually handled by DSU developers.

After running the target method on two programs, our tool will also check the behavior consistency of return values and thrown 
exceptions, and report the result to DSU developers for their further review of state consistency.

\subsection{Transformation Simulation}

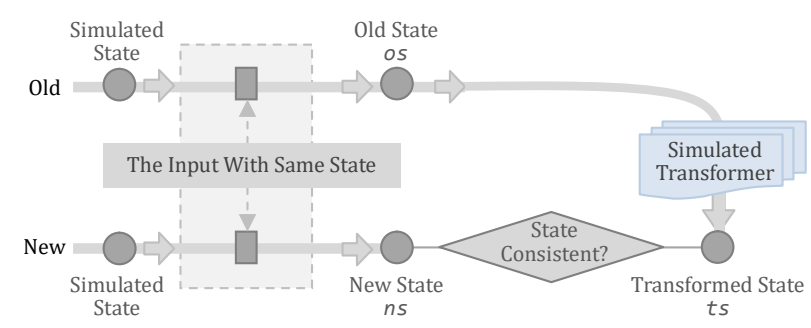

Figure 5: Transformation Simulation

To avoid potential crashes and additional time costs, we simulate the transformation process instead of calling the real DSU. Basically, an object transformer is a special method, working on the old code and constructing the values for new fields. Our simulated transformers mimic this process. There are many approaches to realize the transformation process, but we choose the simple way which require less implementation.

As shown in Fig. 5, we can obtain the object states (os and ns) after running the target method on two version programs, then obey the following rules to simulate the transformation:

1) If no transformer is specified by DSU developers, our tool will perform the default transformation for the old state (os). Default transformation will copy the values for all unchanged fields and assign default values for those changed fields, then the transformed state (ts) can be obtained.

2) Otherwise, our tool will transform the old state according to the rules specified by DSU developers.

\subsection{Consistency Checking}

Our tool checks the consistency between two version programs after running the target method. As shown in Fig. 5, we compare the state consistency of the new state and the transformed state (ns and ts) to check the consistency. Our tool provides a default comparator to finish the checking, which forms a strict comparison requiring the equivalence of each couple of the nodes shown in Fig. 2. However, DSU developers sometimes need a flexible rule for the state comparison. For example, we allow the approximate equality of double values when testing the financial systems.

Therefore, our tool provides flexible choices for DSU developers to check the state consistency:

a) each couple of nodes should be equivalent (default);

b) each couple of nodes in the given depth should be equivalent (to avoid infinite loops);

c) fit the rules that DSU developers manually specify.

Then the consistency checking can be customized based on the DSU requirements.

\subsection{Fuzzing and Efficiency Improvement}

The initial seed pool can be provided manually, which has a great impact on the performance of the fuzzing process. Besides, the original

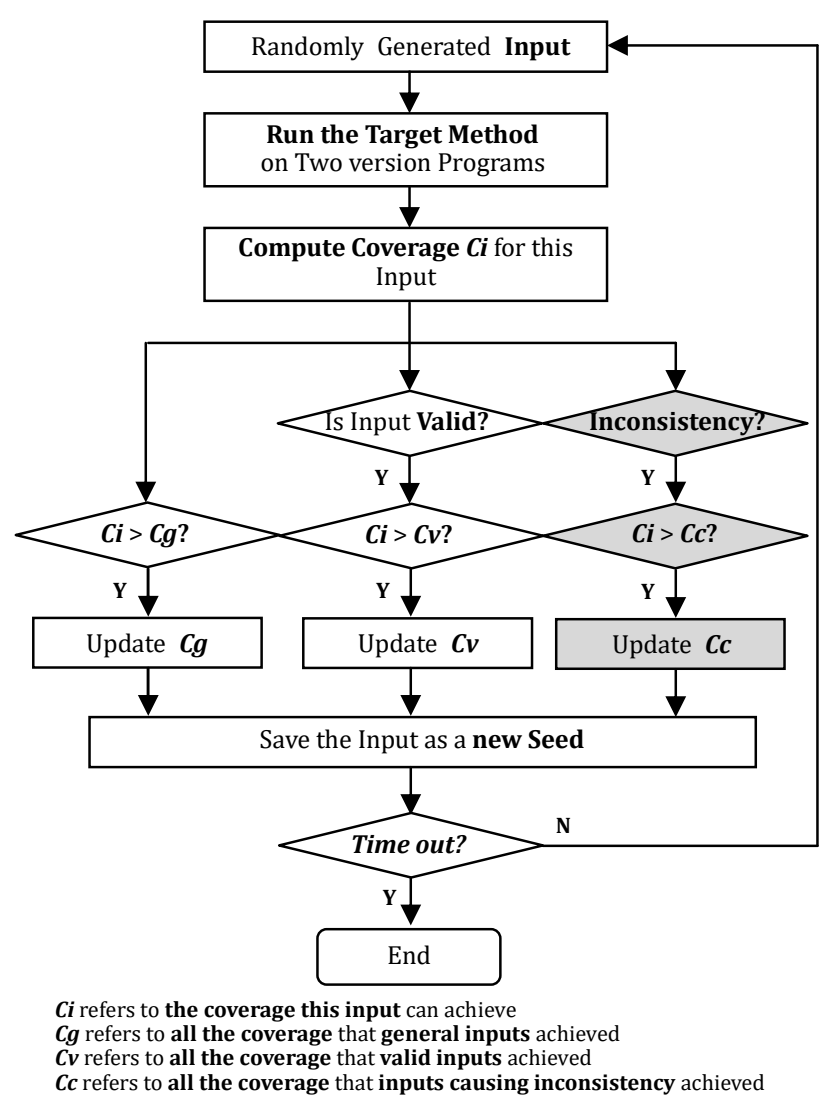

Figure 6: The Strategy for Seed Selection

guidance employs two types of coverage: the general-input coverage and the valid-input coverage. In our work, we add a consistencyinput coverage for further guidance. All the coverage stores the branches that one input can achieve, and we will discuss it later.

We implement our tool based on an open-source fuzzer $\mathcal{F Q F -}$ Zest $[18,19]$, which provides a basic framework for fuzzing testing for Java programs. We make the following modifications to the fuzzer:

1) The original fuzzer only provides the fuzzing framework, not supporting two-version execution, transformation simulation, consistency checking, or the automatic generation of state builder. In our work, we realize these functions to handle DSU problems.

2) The original fuzzer employs the guidance making use of two types of coverage, which store the branches that general inputs and valid inputs can separately achieve. Our tool adds a consistency coverage to store the branches that those special inputs (which cause the inconsistency) can achieve.

There are several key points in the fuzzing testing:

a) How does the fuzzer work

At each run, the fuzzer firstly generates the input parameters of random states by using the seeds and state builders. Then, it calls parallel executor to run the target method on two version programs. After that, the fuzzer calls the transformation simulator and checks the consistency. If the consistency remains, it will report 
$\mathrm{OK}$ and start the next run. Otherwise, it will report the root cause and the state of input parameters to DSU developers, indicating an inconsistency found in the test.

b) How to exclude duplicate inputs

Our tool will print the input states if it finds any inconsistency. However, the fuzzer may generate many duplicate inputs, which are useless and can trigger the same inconsistency many times. For instance, an integer of value $\theta$ will cause an inconsistency between two programs. Each time the fuzzer generates the value 0 , our tool will report a new inconsistency, which is meaningless and trivial.

To solve this problem, our tool obtains the value of each node (see Fig. 2), serializes the inputs as a String, and excludes duplicate inputs. It is not time-consuming since the duplication should be rare if we use a valid state builder.

\section{c) How does the guidance work}

The fuzzer generates the input based on seeds [3], which are the minimized savings of all random results. If an input causes the inconsistency, the fuzzing guidance will compute the coverage this input can achieve, then compare it to the old coverage recorded at the end of the last run. Once any new coverage is found, the input will be saved as a new seed, and the coverage record will be updated.

\section{d) How does our work improve the efficiency}

As shown in Fig. 6, the original fuzzer employs the guidance based on two types of coverage. The first one is general-input coverage, it stores the branches that general inputs can achieve. When an input achieves more coverage than before, it will be saved as a new seed and the coverage recording will be updated. The second one is valid-input coverage, which functions similarly with the general-input coverage, but the inputs must pass the validity checking provided by JQF-Zest [19].

However, the old guidance may not suit DSU problems. Assume that a valid input causes inconsistency between two programs, but its coverage is not larger than $C g$ and $C v$, then this input will not be saved as a new seed. As a result, the following fuzzing process will be inefficient since the input generation is validity-oriented.

Therefore, we add a new consistency-input coverage to make full use of the feedback (Fig. 6). When an input causes the inconsistency, our tool will compare its coverage with saved $C c$ to decide whether this input should be saved. By using the new guidance instead, our tool can save more interesting inputs, and generate more inputs that can trigger the state inconsistency.

\section{EXPERIMENTAL EVALUATION}

In the evaluation of our approach, we mainly want to answer the following questions. First, the effectiveness of our approach, i.e., whether our approach can find any inconsistency in updates. Second, the efficiency of the new coverage guidance, i.e., does the new guidance make the fuzzing process run faster in generating valid inputs that can trigger the inconsistency.

\subsection{Subjects}

We choose a real-world server program, Tomcat, as our experiment subject. Server programs have to provide continuous services, therefore DSU should be integrated into their scenarios. Tomcat is a large-scale open-source project that undergoing long-time maintenance. Therefore, we can find many updates from its development history.

We choose Tomcat 8 as the experiment subject. There are totally 2,114 commits between the version 8.0.0 and the version 8.0.53. We focus on a special set of changes, i.e. the minor changes in the method body since they will be easily ignored by DSU developers. We find 1,149 commits only modifying the method rather than the field. We follow these rules to choose 50 commits in chronological order:

- The change is valid and meaningful, excluding the change to test cases, configuration files, and comments.

- The change only occurs in the method body, excluding changing fields, adding or removing methods.

- The change should be local, excluding changing hierarchies.

- The execution of the changed method does not involve any configuration files or network connections, since we can hardly simulate their states.

In our experiment, we only check the inconsistency of the default transformer, since it can be generated automatically by most DSU systems, and it may introduce more potential errors than manuallyprepared transformation.

\subsection{Experiments}

The seed pool in our tool is initially empty, thus the original inputs are generated randomly. State builder is generated automatically by default, but we manually make some improvements. We extend the range of randomness in our state builder to verify the efficiency of our tool. Besides, some special cases can only be handled manually. For example, it is common in server programs that a method receives a String as the input, which should conform with the URL format. For that update, we have to customize the state builder to generate valid URL strings as the input parameters.

For each commit, we validate our approach from two aspects: effectiveness and efficiency. To validate the effectiveness of our tool, we firstly give the ground truth by manual verification. Then, we execute the approach for 1 hour on each update, record the test results and compare them with the ground truth.

To evaluate the efficiency of our approach, we run the test separately with original fuzzing guidance and with new fuzzing guidance. Each run lasts for 1 hour, and we record the following data every 5 seconds:

- $I_{\text {total }}$ : The number of all generated inputs.

- $I_{\text {valid }}$ : The number of the inputs which are valid (passing the validity checking provided by JQF-Zest).

- IC $C_{\text {origin }}$ : The number of valid inputs which cause the inconsistency with original fuzzing guidance.

- $I C_{n e w}$ : The number of valid inputs which cause the inconsistency with new fuzzing guidance.

\subsection{Results}

4.3.1 Effectiveness. We check the consistency with some valid heuristics, which may introduce some false positives and false negatives since the test results may not fit our expectations semantically. Therefore, we firstly give the ground truth to specify whether the states after the default transformation is semantically consistent 


\begin{tabular}{|c|c|c|c|c|c|c|c|c|c|c|c|}
\hline \multirow{2}{*}{ ID } & \multirow{2}{*}{ Subject } & \multicolumn{2}{|c|}{ State Consistency } & \multicolumn{2}{|c|}{ Behavior Consistency } & \multirow{2}{*}{ ID } & \multirow{2}{*}{ Subject } & \multicolumn{2}{|c|}{ State Consistency } & \multicolumn{2}{|c|}{ Behavior Consistency } \\
\hline & & Manual & Approach & Return & Exception & & & Manual & Approach & Return & Exception \\
\hline 1 & 5263f2-HttpParser & $\checkmark$ & $\sqrt{ }$ & $\sqrt{ }$ & $\sqrt{ }$ & 26 & c53809-Cache & $\sqrt{ }$ & $\sqrt{ }$ & $x$ & $\sqrt{ }$ \\
\hline 2 & 43889c-CharChunk & $x$ & $\sqrt{ }$ & $x$ & $\sqrt{ }$ & 27 & 654e51-LegacyCookieProces sor & $x$ & $x$ & $\sqrt{ }$ & $\sqrt{ }$ \\
\hline 3 & 2968e2-Urlutil & $\sqrt{ }$ & $x$ & $x$ & $\sqrt{ }$ & 28 & 7a2eb9-JNDIRealm & $x$ & $x$ & $\sqrt{ }$ & $\sqrt{ }$ \\
\hline 4 & 36a768-DefaultServlet & $\sqrt{ }$ & $\sqrt{ }$ & $\sqrt{ }$ & $\sqrt{ }$ & 29 & 3b165b-ApplicationHttpRequest & $\sqrt{ }$ & $\sqrt{ }$ & $\sqrt{ }$ & $\sqrt{ }$ \\
\hline 5 & e49d1f-HexUtils & $\sqrt{ }$ & $\sqrt{ }$ & $\sqrt{ }$ & $\sqrt{ }$ & 30 & a2daab-DirResourceset & $\sqrt{ }$ & $\sqrt{ }$ & $\sqrt{ }$ & $\sqrt{ }$ \\
\hline 6 & 184703-Cache & $\sqrt{ }$ & $\sqrt{ }$ & $x$ & $\sqrt{ }$ & 31 & 74b553-Corsfilter & $\sqrt{ }$ & $\sqrt{ }$ & $x$ & $\sqrt{ }$ \\
\hline 7 & 86edd3-RequestInfo & $\sqrt{ }$ & $\sqrt{ }$ & $x$ & $\sqrt{ }$ & 32 & $9 c c 600$-RewriteValve & $x$ & $x$ & $\sqrt{ }$ & $\sqrt{ }$ \\
\hline 8 & 3bd031-CachedResource & $\sqrt{ }$ & $\sqrt{ }$ & $\sqrt{ }$ & $\sqrt{ }$ & 33 & cdb327-OutputBuffer & $x$ & $x$ & $\sqrt{ }$ & $\sqrt{ }$ \\
\hline 9 & ce087d-AsyncStateMachine & $x$ & $\sqrt{ }$ & $\sqrt{ }$ & $\sqrt{ }$ & 34 & 69ea86-MessageBytes & $\sqrt{ }$ & $\sqrt{ }$ & $\sqrt{ }$ & $\sqrt{ }$ \\
\hline 10 & f8c8f3-BaseNCodec & $\sqrt{ }$ & $\sqrt{ }$ & $\sqrt{ }$ & $\sqrt{ }$ & 35 & f1add7-ELSupport & $\sqrt{ }$ & $\sqrt{ }$ & $\sqrt{ }$ & $x$ \\
\hline 11 & 9cb624-ErrorPage & $\sqrt{ }$ & $x$ & v & $x$ & 36 & 5731ab-UpgradeUtil & $\sqrt{ }$ & $\sqrt{ }$ & $\sqrt{ }$ & $\sqrt{ }$ \\
\hline 12 & 082fb2-LegacyCookieProcessor & $x$ & $x$ & $\sqrt{ }$ & $\sqrt{ }$ & 37 & 93dd22-HexUtils & $\sqrt{ }$ & $\sqrt{ }$ & $\sqrt{ }$ & $\sqrt{ }$ \\
\hline 13 & $961 \mathrm{fb} 5-T 1 \mathrm{dSc}$ anner & 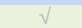 & $\sqrt{ }$ & $\sqrt{ }$ & $\sqrt{ }$ & 38 & b4b8ea-HexUtils & $\sqrt{ }$ & $\sqrt{ }$ & $\sqrt{ }$ & $\sqrt{ }$ \\
\hline 14 & b721c5-RequestUtil & $\sqrt{ }$ & $\sqrt{ }$ & $x$ & $\sqrt{ }$ & 39 & 291eec-Cookie & $x$ & $x$ & $\sqrt{ }$ & $\sqrt{ }$ \\
\hline 15 & c924bc-ServerCookie & $\sqrt{ }$ & $\sqrt{ }$ & $\sqrt{ }$ & $\sqrt{ }$ & 40 & fd3a4e-ServerCookies & $\sqrt{ }$ & $\sqrt{ }$ & $\sqrt{ }$ & $\sqrt{ }$ \\
\hline 16 & 39fb1b-RewriteValve & $x$ & $x$ & $\sqrt{ }$ & $\sqrt{ }$ & 41 & cb3616-Cache & $x$ & $x$ & $\sqrt{ }$ & $\sqrt{ }$ \\
\hline 17 & 387925-Connector & $\sqrt{ }$ & $\sqrt{ }$ & $\sqrt{ }$ & $\sqrt{ }$ & 42 & $9 c c 7 d 2-W e b X m 1$ & $\checkmark$ & $x$ & $\sqrt{ }$ & $\sqrt{ }$ \\
\hline 18 & f6e5d3-MessageBytes & $x$ & $x$ & $\sqrt{ }$ & $\sqrt{ }$ & 43 & c3e254-AstLiteralExpression & $\sqrt{ }$ & $x$ & $\sqrt{ }$ & $\sqrt{ }$ \\
\hline 19 & 35f884-ApplicationHttpRequest & $\sqrt{ }$ & $\sqrt{ }$ & $\sqrt{ }$ & $\sqrt{ }$ & 44 & c46aa5-ELParser & $\sqrt{ }$ & $\sqrt{ }$ & $\sqrt{ }$ & $\sqrt{ }$ \\
\hline 20 & 5816ff-ReplicatedMap & $\sqrt{ }$ & $\sqrt{ }$ & $\sqrt{ }$ & $\sqrt{ }$ & 45 & d49a03-ChunkedInputFilter & $\sqrt{ }$ & $\sqrt{ }$ & $\sqrt{ }$ & $\sqrt{ }$ \\
\hline 21 & c79b55-UniqueID & $\sqrt{ }$ & $\sqrt{ }$ & $\sqrt{ }$ & $\sqrt{ }$ & 46 & fc6db3-Response & $x$ & $x$ & $\sqrt{ }$ & $\sqrt{ }$ \\
\hline 22 & 90a86f-Util & $\sqrt{ }$ & $\sqrt{ }$ & $\sqrt{ }$ & $\sqrt{ }$ & 47 & a49762-VoidInputFilter & $x$ & $\sqrt{ }$ & $x$ & $\sqrt{ }$ \\
\hline 23 & 016647-HttpMessages & $\sqrt{ }$ & $\sqrt{ }$ & $\sqrt{ }$ & $\sqrt{ }$ & 48 & 6 de 83 c-Streams & $\sqrt{ }$ & $\sqrt{ }$ & $\sqrt{ }$ & $\sqrt{ }$ \\
\hline 24 & 50f017-RequestUtil & v & $\sqrt{ }$ & $x$ & $\sqrt{ }$ & 49 & 4e4ae4-Request & $x$ & $x$ & $\sqrt{ }$ & $\sqrt{ }$ \\
\hline 25 & 157cc7-Util & $\sqrt{ }$ & $\sqrt{ }$ & $\sqrt{ }$ & $\sqrt{ }$ & 50 & 937760-ExpressionTokenizer & $\sqrt{ }$ & $x$ & $\sqrt{ }$ & $\sqrt{ }$ \\
\hline
\end{tabular}

Figure 7: Experiment Result of Effectiveness

with the new code. Then, compare the test results with the ground truth to prove the validity of our heuristics.

Figure 7 shows the detailed results. The subject column shows the commit id of the update and the name of the target class. Green cells and blue cells mean our approach fits our manual results. The blue one indicates an inconsistency problem is found and the green one does not. Red cells mean the results of our approach are different from manual results. In the 50 updates, 14 of them have the inconsistency problem while the other 36 do not. Our approach detected the inconsistency in 16 updates, including 5 false positives and 3 false negatives.

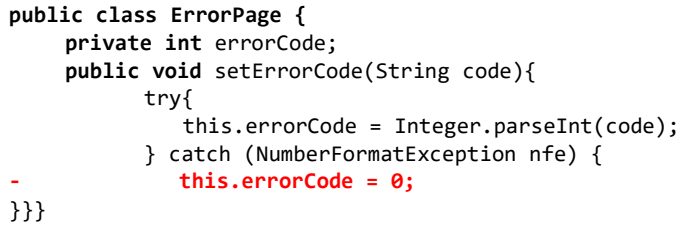

Figure 8: Snippets in commit 9cb624

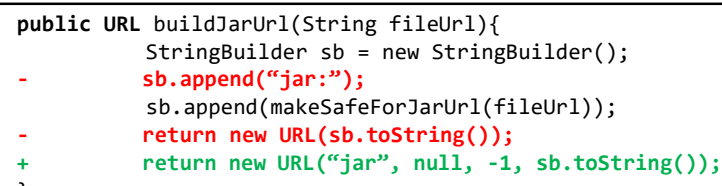

Figure 9: Snippets in Commit 2968e2

\section{a) False Positive}

Two reasons will cause a false positive. One is those randomly generated inputs, which actually cause the inconsistency, are practically meaningless.
As shown in Fig. 8, the testing process first initializes the field errorCode with a given value (usually non-zero) and calls setErrorCode method to change its value. In the old version program, when the input String can not conform to the Number format, the errorCode will be set to $\theta$, whereas in the new version, the value of errorCode remains unchanged. Applying default transformer on the old version object will copy value 0 , which will definitely cause an inconsistency with the new version program. However, this kind of inputs is meaningless, since the inputs in practice should be semantically valid and will not throw exceptions.

The other reason is that the inconsistency itself is practically meaningless. Commit $2968 \mathrm{e} 2$ changes the way to create an URL link. We think this update will not have an inconsistency problem since it only involves some optimizations in memory consumption. However, our approach actually detects the state inconsistency in the field, host, inside the URL object: the field value in the old version is empty while in new is null. Our tool reports this state inconsistency to developers. After manual examination, this update will not cause any semantic inconsistency, which can be transformed by default transformer.

These false positives will not cause serious security problems for an update, since our approach reports all inconsistencies for DSU developers to review manually.

\section{b) False Negative}

Two reasons can cause a false negative. Firstly, our tool failed to detect some inconsistencies due to implementation limits. Moreover, our tool fails to detect systemic inconsistencies, since we simplify the problem to the unit testing. Commit a49762 changes a simple method that returns the availability of an input filter. This input filter validates the inputs for many requests which travel between components in the whole server program. Thus, their inconsistent states will cause a huge influence on global state consistency. Our tool fails to detect such kind of inconsistencies.

These false negatives may cause great security problems if developers ignore the potential inconsistencies. As shown in Fig. 11, our 


\begin{tabular}{|c|c|c|c|c|c|c|c|c|c|c|c|c|c|c|c|c|c|}
\hline \multirow{2}{*}{ ID } & \multicolumn{2}{|c|}{$\begin{array}{l}\text { With new } \\
\text { guidance }\end{array}$} & \multicolumn{2}{|c|}{$\begin{array}{l}\text { With original } \\
\text { guidance }\end{array}$} & \multirow{2}{*}{$\begin{array}{l}\text { Trend } \\
\text { Chart }\end{array}$} & \multirow{2}{*}{ ID } & \multicolumn{2}{|c|}{$\begin{array}{l}\text { With new } \\
\text { guidance }\end{array}$} & \multicolumn{2}{|c|}{$\begin{array}{l}\text { With original } \\
\text { guidance }\end{array}$} & \multirow{2}{*}{$\begin{array}{l}\text { Trend } \\
\text { Chart }\end{array}$} & \multirow{2}{*}{ ID } & \multicolumn{2}{|c|}{$\begin{array}{l}\text { With new } \\
\text { guidance }\end{array}$} & \multicolumn{2}{|c|}{$\begin{array}{l}\text { With original } \\
\text { guidance }\end{array}$} & \multirow{2}{*}{$\begin{array}{l}\text { Trend } \\
\text { Chart }\end{array}$} \\
\hline & Ivalid & ICnew & Ivalid & ICorigin & & & Ivalid & ICnew & Ivalid & ICorigin & & & Ivalid & ICnew & Ivalid & ICorigin & \\
\hline 3 & 122337 & 122337 & 102074 & 102074 & & 28 & 283190 & 1118 & 279750 & 553 & & 43 & 311184 & 69641 & 305551 & 25724 & $\sim$ \\
\hline 11 & 173745 & 8786 & 183101 & 4643 & & 32 & 66301 & 9214 & 66844 & 7174 & & 46 & 67681 & 2 & 69164 & 2 & 5 \\
\hline 12 & 3 & 3 & 3 & 3 & & 33 & 16021 & 618 & 16973 & 191 & & 49 & 16204 & 342 & 34647 & 218 & \\
\hline 16 & 84998 & 7346 & 85372 & 5058 & & 39 & 37945 & 4186 & 39713 & 3139 & & 50 & 202600 & 12386 & 214091 & 1214 & \\
\hline 18 & 191234 & 191234 & 193749 & 193749 & & 41 & 160628 & 17100 & 160168 & 11024 & & & & & & & \\
\hline 27 & 178803 & 2 & 181408 & 2 & & 42 & 88064 & 16956 & 88034 & 15079 & & & & & & & \\
\hline
\end{tabular}

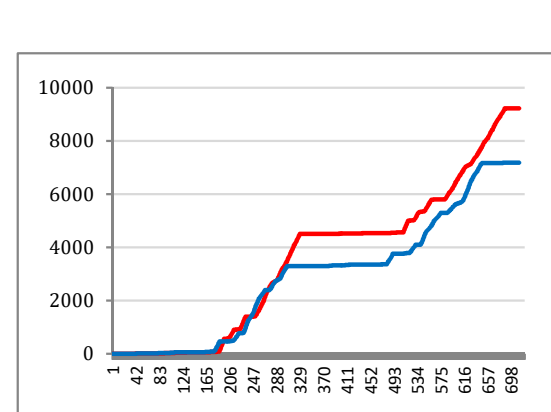

a) Update ID.32

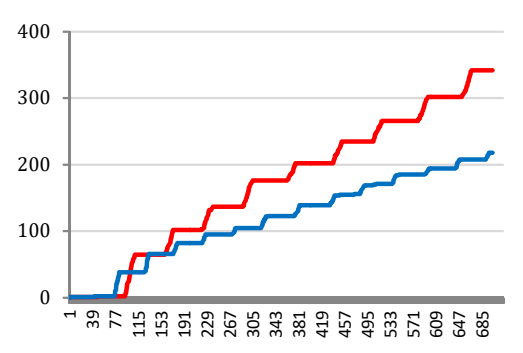

b) Update ID.49

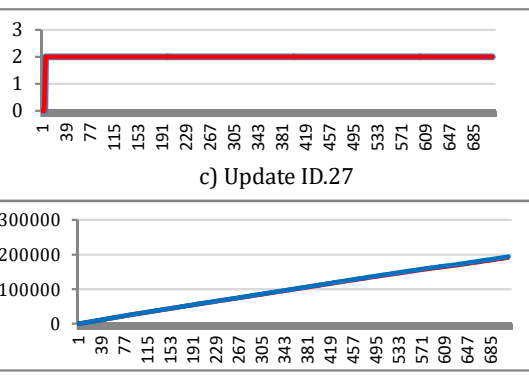

d) Update ID.18

The unit of abscissa is per five seconds.

Figure 10: $I C_{\text {origin }}$ and $I C_{n e w}$ of 16 Updates

tool also presents some additional information for DSU developers to review them manually, such as all generated inputs and the consistency of return values and thrown exceptions. Developers may find some interesting ones when preparing a better object transformer.

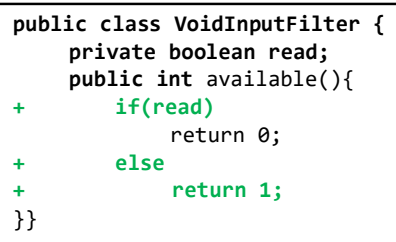

Figure 11: Snippets in Commit a49762

4.3.2 Efficiency. We run our approach separately with original fuzzing guidance and the new guidance on those 16 updates that our approach actually detects the inconsistency. As shown in Fig. 12, with the new guidance, 164,711 valid inputs are generated on average for each update, and 45.75 valid inputs are generated per second, which is similar to the result running with original guidance (168,590 valid inputs in total and 46.83 valid inputs per second). However, with the new coverage guidance, more inputs that can trigger the inconsistency are generated. Our tool generates 28,243 special inputs totally and generates about 7.85 special inputs per second, while the original fuzzer generates about 6.28 special inputs per second. The efficiency is increased by $25.0 \%$.

The results of 16 updates are shown in Fig. 10. Among these updates, 12 updates show great efficiency improvement with the new guidance. 3 updates find few inconsistencies. 1 update can not show great improvement.

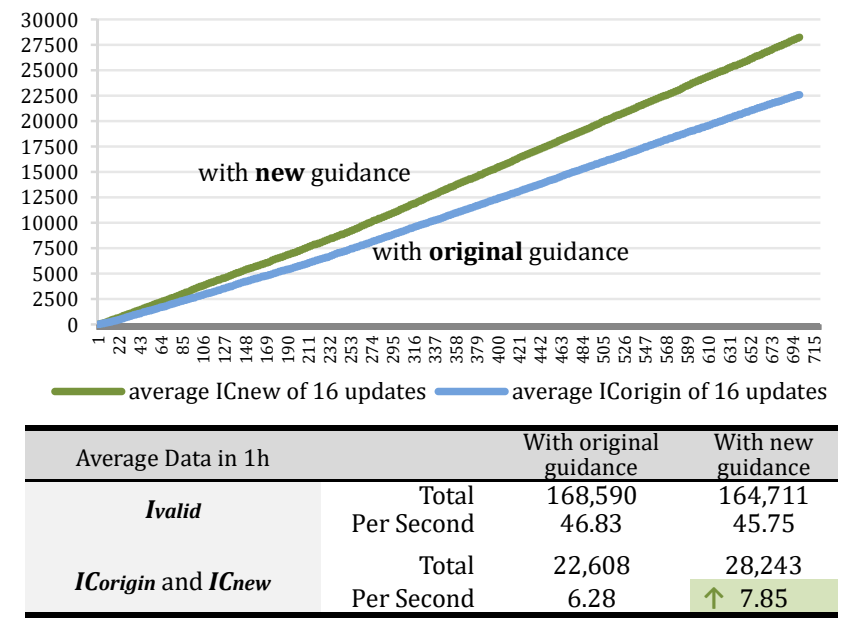

Figure 12: Overview of Efficiency

a) 12 updates show great improvement with new guidance since we add a new consistency coverage to guide the seed selection. For example, the results of ID. 32 and ID. 49 updates are typical enough to prove the advantage. $I C_{n e w}$ is smaller than $I C_{\text {origin }}$ at the beginning of the test, but it increases more sharply since our tool selects the seeds with high quality, able to generate more expected inputs causing the inconsistency.

b) 3 updates find few inconsistencies. For example, an inconsistency may be triggered by some particular inputs. Our tool can exclude duplicate inputs to avoid the explosion of result reporting.

c) 1 update can not show great improvement, since the inconsistency can be easily triggered. The strategy of seed selection can greatly influence the range where the fuzzer is searching, but it 
will lose its effect if any randomly generated input can cause the inconsistency.

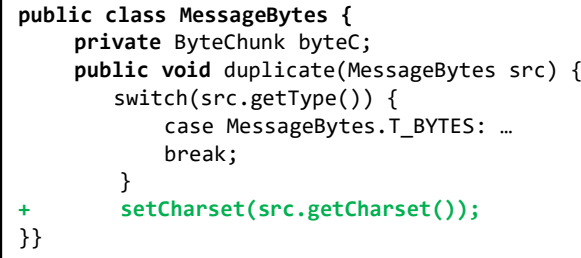

Figure 13: Snippets in Commit f6e $5 \mathrm{~d} 3$

Taking ID. 18 update as an example, the method duplicate of the new program will modify the field bytec. Therefore, almost all valid inputs can lead to the different states of bytec, easily causing the inconsistency. In that case, the inputs generated with guidance will not perform more efficiently than those generated with complete randomness.

The previous results show that our approach can effectively and efficiently examine the inconsistency problem of the default transformer, and we believe our approach can also be applied to more complicated transformers.

\section{DISCUSSION}

We make several assumptions to simplify the problem. Firstly, our approach works as the unit testing by simulating the state of inputs. It will be useful when a minor modification occurs in a single method. However, if many methods are changed at the same time, DSU developers can run the test separately on each modified method. In our experiment, we only focus on the changes in a single method to validate the basic effectiveness of our approach.

The simulation of object states will inevitably result in many false warnings. For instance, a URL causing the inconsistency between two version programs may be practically meaningless. We report all of them for DSU developers to review manually, but we should propose a better strategy to exclude these meaningless inputs in future work.

\section{RELATED WORK}

There are many existing DSU systems. Dynamic Code Evolution (DCE VM) [26] supports a variety of updates, e.g. the change to methods or fields. Jvolve [23] does not support binary incompatible changes, like deleting fields or methods. Javelus [10] supports all kinds of updates and is more compatible than Jvolve. To update a running program, default transformers can be used directly, or else developers have to provide a complicated transformer manually. Our work is orthogonal to these DSU systems.

The default transformer provides a trivial way to transform the object states. It only copies the value for unchanged fields and assigns default values for newly added fields. TOS [17] is a programming-by-example approach that takes old and new version objects as input and synthesizes object transformers from a set of predefined rules. AOTES [11] first synthesizes a method history that can create the current state of a stale object, then replays the history on the new version program to create the corresponding new version object. Our approach is complementary to these transformers since our approach can improve their reliability.

\section{CONCLUSION AND FUTURE WORK}

In this paper, we propose a testing approach for DSU programs to help developers improve the reliability of the object state transformer. We provide the state builder to simulate object states, call parallel executor to run the target method on two versions of the program, simulate the transformation process, and then employ the fuzzing testing with new guidance to generate test inputs efficiently. As a result, we validate the effectiveness and efficiency of our approach.

In future work, we will keep improving our approach and tool implementations, and try to solve the problems mentioned in section 5. For example, we can run the target methods in a certain order instead of a single method. Besides, we should propose a better strategy to exclude those practically meaningless inputs.

\section{ACKNOWLEDGMENTS}

The authors would like to thank the anonymous reviewers for their suggestions. This work is supported by the Key-Area Research and Development Program of Guangdong Province (NO.2020B010164003) and the National Natural Science Foundation of China (Grant 62025202).

\section{REFERENCES}

[1] 1998. Differential Testing for Software. Differential Testing for Software. (1998)

[2] 2003. Apache Tomcat. http://tomcat.apache.org/. Accessed: 2020-07-20.

[3] 2014. Optimizing seed selection for fuzzing. In Proceedings of the 23rd USENIX Security Symposium.

[4] 2020. Hydiff: Hybrid differential software analysis. https://doi.org/10.1145/ 3377811.3380363

[5] Marcel Bohme, Van Thuan Pham, and Abhik Roychoudhury. 2019. CoverageBased Greybox Fuzzing as Markov Chain. IEEE Transactions on Software Engineering (2019). https://doi.org/10.1109/TSE.2017.2785841

[6] Haipeng Cai, Siyuan Jiang, Raul Santelices, Ying Jie Zhang, and Yiji Zhang. 2014. SENSA: Sensitivity analysis for quantitative change-impact prediction. In Proceedings - 2014 14th IEEE International Working Conference on Source Code Analysis and Manipulation, SCAM 2014. https://doi.org/10.1109/SCAM.2014.25

[7] Haipeng Cai and Raul Santelices. 2015. A comprehensive study of the predictive accuracy of dynamic change-impact analysis. In fournal of Systems and Software. https://doi.org/10.1016/j.jss.2015.02.018

[8] Yuting Chen, Ting Su, Chengnian Sun, Zhendong Su, and Jianjun Zhao. 2016. Coverage-Directed differential testing of JVM implementations. In Proceedings of the ACM SIGPLAN Conference on Programming Language Design and Implementation (PLDI). https://doi.org/10.1145/2908080.2908095

[9] Shigeru Chiba. 1998. Javassist-A Reflection-based Programming Wizard for Java. Proceedings of OOPSLA'98 Workshop on Reflective Programming in $\mathrm{C}++$ and Java (1998).

[10] Tianxiao Gu, Chun Cao, Chang Xu, Xiaoxing Ma, Linghao Zhang, and Jian Lu. 2012. Javelus: A Low Disruptive Approach to Dynamic Software Updates. In Proceedings of 19th the Asia-Pacific Software Engineering Conference. 527-536.

[11] Tianxiao Gu, Xiaoxing Ma, Chang Xu, Yanyan Jiang, Chun Cao, and Jian Lu. 2018. Automating Object Transformations for Dynamic Software Updating via Online Execution Synthesis. In European Conference on Object-Oriented Programming.

[12] Jianmin Guo, Yu Jiang, Yue Zhao, Quan Chen, and Jiaguang Sun. 2018. DLFuzz: Differential fuzzing testing of deep learning systems. In ESEC/FSE 2018 - Proceedings of the 2018 26th ACM Foint Meeting on European Software Engineering Conference and Symposium on the Foundations of Software Engineering. https://doi.org/10.1145/3236024.3264835 arXiv:1808.09413

[13] Christopher M. Hayden, Edward K. Smith, Eric A. Hardisty, Michael Hicks, and Jeffrey S. Foster. 2012. Evaluating dynamic software update safety using systematic testing. IEEE Transactions on Software Engineering (2012). https: //doi.org/10.1109/TSE.2011.101

[14] Roya Hosseini and Peter Brusilovsky. 2013. JavaParser: A fine-grain concept indexing tool for java problems. In CEUR Workshop Proceedings. 
[15] Xiao Liu, Xiaoting Li, Rupesh Prajapati, and Dinghao Wu. 2019. DeepFuzz: Automatic generation of syntax valid C programs for fuzz testing. In 33rd $A A A I$ Conference on Artificial Intelligence, AAAI 2019, 31st Innovative Applications of Artificial Intelligence Conference, IAAI 2019 and the 9th AAAI Symposium on Educational Advances in Artificial Intelligence, EAAI 2019. https://doi.org/10. 1609/aaai.v33i01.33011044

[16] Xiaoxing Ma, Luciano Baresi, Carlo Ghezzi, Valerio Panzica La Manna, and Jian Lu. 2011. Version-consistent dynamic reconfiguration of component-based distributed systems. https://doi.org/10.1145/2025113.2025148

[17] Stephen Magill, Michael Hicks, Suriya Subramanian, and Kathryn S. McKinley. 2012. Automating Object Transformations for Dynamic Software Updating. In Proceedings of the ACM International Conference on Object Oriented Programming Systems Languages and Applications. 265-280.

[18] Rohan Padhye, Caroline Lemieux, and Koushik Sen. 2019. JQF: Coverage-guided property-based testing in Java. In ISSTA 2019 - Proceedings of the 28th ACM SIGSOFT International Symposium on Software Testing and Analysis. https://doi. org/10.1145/3293882.3339002

[19] Rohan Padhye, Caroline Lemieux, Koushik Sen, Mike Papadakis, and Yves Le Traon. 2019. Semantic fuzzing with ZEST. In ISSTA 2019 - Proceedings of the 28th ACM SIGSOFT International Symposium on Software Testing and Analysis. https://doi.org/10.1145/3293882.3330576 arXiv:1812.00078

[20] Luis Pina and Michael Hicks. 2016. Tedsuto: A General Framework for Testing Dynamic Software Updates. In Proceedings - 2016 IEEE International Conference on Software Testing, Verification and Validation, ICST 2016. https://doi.org/10.
1109/ICST.2016.27

[21] Xiaoxia Ren, Fenil Shah, Frank Tip, Barbara G Ryder, and Ophelia Chesley. 2004. Chianti: A Tool for Change Impact Analysis of Java Programs. Proceeding OOPSLA '04 Proceedings of the 19th annual ACM SIGPLAN conference on Object-oriented programming, systems, languages, and applications (2004). https://doi.org/10. $1145 / 1035292.1029012$

[22] Suriya Subramanian, Michael Hicks, and Kathryn S. McKinley. 2009. Dynamic software updates: A VM-centric approach. In Proceedings of the ACM SIGPLAN Conference on Programming Language Design and Implementation. 1-12.

[23] Suriya Subramanian, Michael Hicks, and Kathryn S. McKinley. 2009. Dynamic software updates: A VM-centric approach. In Proceedings of the ACM SIGPLAN Conference on Programming Language Design and Implementation (PLDI). https: //doi.org/10.1145/1542476.1542478

[24] William N. Sumner and Xiangyu Zhang. 2013. Comparative causality: Explaining the differences between executions. In Proceedings - International Conference on Software Engineering. https://doi.org/10.1109/ICSE.2013.6606573

[25] Chenxin Wang and Shunyao Kang. 2018. ADFL: An improved algorithm for american fuzzy lop in fuzz testing. In Lecture Notes in Computer Science (includin subseries Lecture Notes in Artificial Intelligence and Lecture Notes in Bioinformatics). https://doi.org/10.1007/978-3-030-00018-9_3

[26] Thomas Würthinger, Christian Wimmer, and Lukas Stadler. 2010. Dynamic code evolution for Java. In Proceedings of the 8th International Conference on the Principles and Practice of Programming in Java, PPPf 2010. https://doi.org/10. $1145 / 1852761.1852764$ 